Research Paper

\title{
Metronomic vinorelbine is an excellent and safe treatment for advanced breast cancer: a retrospective, observational study
}

Chien-Ting Liu ${ }^{1,2^{*}}$, Meng-Che Hsieh ${ }^{3,4^{*}}$, Yu-Li Su ${ }^{1,2}$, Chaio-Ming Hung ${ }^{4,5}$, Sung-Nan Pei ${ }^{3,4}$, Chun-Kai Liao1, Yu-Fen Tsai ${ }^{3,4}$, Hsiu-Yun Liao ${ }^{3}$, Wei-Ching Liu'3 , Chong-Chi Chiu 4,5, Shih-Chung Wu ${ }^{6}$, Shih-Ho Wang2,6, Ching-Ting Wei ${ }^{7}$, Kun-Ming Rau ${ }^{3,4^{凶}}$

1. Division of Hematology-Oncology, Department of Internal Medicine, Kaohsiung Chang Gung Memorial Hospital, Kaohsiung 833, Taiwan.

2. Chang Gung University, College of Medicine, Tao-Yuan 333, Taiwan

3. Department of Hematology-Oncology, E-Da Cancer Hospital, Kaohsiung 822, Taiwan.

4. College of Medicine, I-Shou University, Kaohsiung 822, Taiwan.

5. Department of Surgery, E-Da Cancer Hospital, Kaohsiung 822, Taiwan.

6. Department of Surgery, Kaohsiung Chang Gung Memorial Hospital, Kaohsiung 833, Taiwan.

7. Division of General Surgery, Department of Surgery, E-Da Hospital, Kaohsiung, 822 Taiwan.

* Equally contribution.

$\triangle$ Corresponding author: Kun-Ming Rau, MD, liu07822@ms57.hinet.net, Tel.: +886-7-6150022

(1) The author(s). This is an open access article distributed under the terms of the Creative Commons Attribution License (https://creativecommons.org/licenses/by/4.0/). See http://ivyspring.com/terms for full terms and conditions.

Received: 2021.03.20; Accepted: 2021.06.20; Published: 2021.07.03

\begin{abstract}
Advanced breast cancer ( $A B C)$ has become a chronic disease. In such a situation, an effective therapy with low toxicities and economically acceptable is needed. Metronomic vinorelbine (mVNR) has been proved to be effective on the control of MBC. The aim of this study is to evaluate the efficacy and safety of $m V N R$ as the salvage therapy for patients with $A B C$. Oral vinorelbine (VNR) was administered at 70 $\mathrm{mg} / \mathrm{m} 2$, fractionated on days 1,3 , and 5 , for 3 weeks on and 1 week off. Once the $\mathrm{mVNR}$ was combined with trastuzumab, or was combined with bevacizumab, the schedule was changed to 2 weeks on and 1 week off. Clinical data of patients with $A B C$ who had received treatment with mVNR and tumor characteristics were collected and analyzed. From Mar. 2013 to Dec, 2020, there were 90 patients with $A B C$ received $m V N R$. The overall response rate was $53.3 \%$ and overall disease control rate (DCR) was $78.9 \%$ in this study, including 4 (4.4\%) cases reached complete response, 44 (48.9\%) cases reached partial response and $23(25.6 \%)$ cases were table disease. The median time to treatment failure (TTF) of the Lumina A patients was 13.3 months, Lumina B patients was 9.1 months, Her-2 enrich patients was 8.9 months, and triple negative breast cancer (TNBC) patients was 5.6 months. Median overall survival time for Lumina A, Lumina B, Her-2 enrich and TNBC were 54.6 months, 53.3 months, 59.5 months and 24.5 months separately. Side effects were minimal and manageable. Metronomic VNR can be an effective treatment for $A B C$ either works as a switch maintenance or salvage therapy. In combination with target therapy or hormonal therapy, mVNR can further improve TTF and DCR with minimal toxicities. Further study should focus on the optimal dosage, schedule and combination regimen.
\end{abstract}

Key words: metastatic breast cancer, advanced breast cancer, metronomic chemotherapy, vinorelbine, effect.

\section{Introduction}

Advanced breast cancer (ABC), including metastatic breast cancer (MBC) and locally advanced breast cancer (LABC) are generally incurable diseases. However, because of improvements in systemic therapies, the overall survival time of $\mathrm{ABC}$ patients is getting longer. As a palliative setting for $A B C$, good control of tumors, economically acceptable for the healthcare system, and avoidance of toxicity from therapies might be as important as improving survival. 
Although recent improvements of hormonal therapies and targeted therapies do provide good tumor control with low toxicity profiles, chemotherapy is still required during the course of treatment in most patients [1]. Conventional chemotherapy administers drugs at, or close to, the maximal tolerated dose (MTD). While MTD chemotherapy might kill chemotherapy-sensitive cancer cell populations, the toxicities may deteriorate patients' quality of life (QoL), so a drug-free interval is needed. A break in therapy, however, may allow resistant cells to re-colonize, ultimately leading to disease progression and development of resistance [2].

In contrast to MTD drug regimens, metronomic chemotherapy (MCT) is the continuous administration of drugs at minimally toxic doses without prolonged drug-free intervals. MCT was first introduced in 2000 by Douglas Hanahan, based on preclinical papers from the laboratories of Judah Folkman and Robert Kerbel $[3,4]$. MCT has an effect on tumor cells, and also the surrounding microenvironment. A fundamental concept of MCT is that tumor cells might acquire resistance to cytotoxic chemotherapy, but acquired resistance would not be expected for vascular endothelial cells [5]. In an early experiment, an anti-angiogenic schedule of cyclophosphamide (CTX) increased the apoptosis of endothelial cells that preceded the apoptosis of drug-resistant tumor cells. This metronomic schedule eradicated the majority of drug-resistant Lewis lung carcinomas, and avoided acquired drug resistance when compared with a conventional chemotherapy schedule [6].

Metronomic administration of chemotherapeutic drugs holds a great deal of promise to address several of the major weak points of MTD regimens. These include the development of drug resistance, suppression of anti-tumor immunity, high toxicity, and poor QoL during therapy. The most suitable agents for MCT are oral, inexpensive, well-tolerated, and with no or minimal cumulative toxicity.

Many studies of MCT have been published over the past decades involving patients with $\mathrm{ABC}$. Experimental protocols have often included CTX, methotrexate, capecitabine (CAPE), and vinorelbine (VNR). These drugs have also been associated with hormonal treatments or targeted agents like trastuzumab and bevacizumab [7]. Based on these advantages, MCT can be an alternative to standard-schedule chemotherapy or as maintenance therapy in times of remission to delay disease progression [8].

Vinorelbine has been approved for the treatment of breast cancer (BC) and non-small cell lung cancer (NSCLC) in Europe, and for NSCLC only in the
United States, alone or in combination with difference chemotherapies [9]. VNR is a semi-synthetic vincaalkaloid that targets tubulin, which prevents tumor cells from making spindles that are needed for division during the cell cycle, and as such has anti-proliferative properties. The recent approval of an oral formulation of VNR has opened the way to development of MCT with this drug. Metronomic VNR (mVNR) had been proven to be well-tolerated, even for elderly patients. Several clinical trials investigating the effect of $\mathrm{mVNR}$ on different kinds of cancer have been performed over the last few years [10]. A phase IA dose-ranging study confirming that mVNR can safely be administered at doses up to 50 mg three time a week and that it can yield not only long-lasting antitumor effect without overt toxicities, but also lack of drug accumulation [11].

In a study of 34 elderly patients not pretreated for MBC, VNR was administered at $70 \mathrm{mg} / \mathrm{m}^{2}$, fractionated on days 1,3 , and 5 , for 3 weeks on and 1 week off, every 4 weeks. The overall response rate (ORR) was 38\%, median progression free survival (PFS) was 7.7 months, and median overall survival (OS) was 15.9 months [12]. For elderly patients with MBC, $30 \mathrm{mg}$ VNR every other day as the first- or further-line treatment, the ORR was $68.7 \%$ including 6 complete response (CR, 18.8\%) and 16 partial response (PR ,50\%). Six patients $(18.7 \%)$ achieved stable disease (SD) with a disease control rate (DCR) of $87.4 \%$. After a median follow-up of 12 months, median PFS was 9.2 months. The most encouraged finding was few grade 3,4 toxicities were reported in these trials [13].

Because most published data regarding $\mathrm{mVNR}$ are from clinical trials, which usually recruit selected patients, the data may not represent real world experience. Thus, in this study we retrospectively collected and analyzed clinical data and treatment outcomes of patients with MBC or LABC who were treated with $\mathrm{mVNR}$, either as a single agent or in combination with other drugs such as hormonal therapy or targeted therapies.

\section{Methods}

\section{Study Population}

Clinical data of patients who received $\mathrm{mVNR}$ between March 2013 and Dec. 2020 at 2 hospitals were retrospectively collected and reviewed. The study design was approved by Institutional Review Board of Kaohsiung Chang Gung Memorial Hospital (IRB No. 201700595B0) and E-Da Cancer Hospital (EMRP-110-040).

Inclusion criteria were pathological confirmed $\mathrm{ABC}$, and treated with mVNR. Tumor characteristics 
such as estrogen receptor (ER), progesterone receptor (PR), and human epidermal growth factor receptor 2 (Her-2) status, Ki-67 expression, and molecular subtype (luminal A, luminal B, Her-2-enriched, and triple-negative breast cancer [TNBC]) were recorded. Metastatic sites including regional lymph nodes and distant organs were also recorded and analyzed.

\section{Treatment}

Vinorelbine was administered at $70 \mathrm{mg} / \mathrm{m}^{2}$, fractionated on days 1,3 , and 5 , for 3 weeks on and 1 week off, every 4 weeks. This schedule was used if VNR was prescribed as a single agent, or was combined with hormonal therapy for hormonal receptor (HR) positive patients. Patients with Her-2 overexpressed breast cancers also received triweekly trastuzumab. If mVNR was combined with trastuzumab for Her-2+ $\mathrm{ABC}$ patients, or was combined with bevacizumab for TNBC patients, the schedule was changed to 2 weeks on and 1 week off, every 3 weeks. Pre-medications included loperamide and prochloperazine, both taken 30 minutes before VNR. Data collected included tumor characteristics, clinical parameters (e.g., site of metastases), treatment events (e.g., number of therapeutic cycles, start/end dates, and rationale for discontinuation), clinical response, use of supportive care medications (e.g., granulocytic colony stimulating factor), dose adjustments, and adverse events. The reported results were based on effectiveness analysis of data collected by Dec. 2020.

\section{Outcome measures}

The primary outcome measure was response rate, including the DCR, which was defined as the proportions of patients who achieved complete response $(\mathrm{CR})$, partial response (PR), and stable disease (SD) as the best response. Tumor response was assessed using Response Evaluation Criteria in Solid Tumors 1.1, with computed tomography scans at baseline. Bone scan and chest X-ray were used as adjuvant evaluation tools. Thus, the percentages of patients with advanced LABC or MBC who achieved $\mathrm{CR}, \mathrm{PR}$, and $\mathrm{SD}$ during $\mathrm{mVNR}$ treatment were recorded. The safety of $\mathrm{mVNR}$ was evaluated by the number of patients with adverse events (AEs) and the severity of AEs, which were assessed using the Common Terminology Criteria for Adverse Events (CTCAE), version 4.0. This included all events that were not present before the initial administration of $\mathrm{mVNR}$, pre-existing events that became more intense or more frequent, and events that were present upon initial $\mathrm{mVNR}$ administration, but became more severe following administration.

\section{Statistical analysis}

Characteristics of patients and tumors, treatment duration, tumor response, and other categorical variables were summarized as number and percentage, and age as median (range). The time to treatment failure (TTF) was defined as the period from the first dose of mVNR to cancellation for any reason including death, disease worsening, treatment toxicity, patient request, or was censored at the date of last follow-up for surviving patients remaining on treatment. Overall survival time (OS) was defined as the period from the first dose of mVNR to the date of patient death, loss of follow-up or the date as of last follow-up for surviving patients. Univariate analysis was performed to determine the associations of tumor responses (objective response rate [ORR], $\mathrm{CR}+\mathrm{PR}$; and $\mathrm{DCR}, \mathrm{CR}+\mathrm{PR}+\mathrm{SD}$ ) with patient characteristics. The differences of tumor responses were compared using the Pearson chi-square test or Fisher's exact test for categorical variables. Subgroup analysis of TTF was performed using the Kaplan-Meier method and logrank test assess the associations of TTF and different characteristics. Univariable Cox regression analyses were also used to identify the associations of TTF and different characteristics. All statistical assessments were 2-tailed, and a value of $p>0.05$ was considered to indicate statistical significance. All data analysis was performed using Stata Statistical Software (Release 11, StataCorp LP, College Station, TX).

\section{Results}

\section{Patient characteristics}

From Mar. 2013 to Dec, 2020, 90 patients with either $\mathrm{LABC}$ or $\mathrm{MBC}$ received $\mathrm{mVNR}$, data of most patients were available for toxicity and effectiveness analysis. A summary of patient characteristics is shown in Table 1.

The median age at the time of starting mVNR was 56.0 years old. There were $24(26.7 \%)$ patients what were luminal A, 39 (43.3\%) were luminal B, 14 (15.6\%) were Her-2 enriched, and $13(14.4 \%)$ patients were TNBC. Forty-three cases $(47.8 \%)$ were HR+, including 19 cases in luminal B, 34 (36.7\%) were Her-2 + , including 20 cases who were HR+ and Her-2 +.

Lung and liver were the most common metastatic organs, and there were $9(10.0 \%)$ patients with only bone metastasis. Most patients had received at least one line of therapy before they began mVNR. The status of switching to mVNR, $45(50 \%)$ patients were PR to the previous regimen, $14(15.6 \%)$ patients were SD. For patients whose previous responses were $\mathrm{CR}$, PR or SD, changing to $\mathrm{mVNR}$ was like switching maintenance therapy. Twenty-six (28.9\%) patients had $\mathrm{PD}$ at the time they switched to mVNR. 
Table 1. Patients baseline characteristics.

\begin{tabular}{|c|c|}
\hline & Number $(\%)$ \\
\hline Median Age at diagnosis, years old (y/o) & 50.0 \\
\hline Median age at the time of starting vinorelbine $(\mathrm{y} / \mathrm{o})$ & 56.0 \\
\hline \multicolumn{2}{|l|}{ Molecular subtype } \\
\hline Lumina A & $24(26.7)$ \\
\hline \multicolumn{2}{|l|}{ Lumina B } \\
\hline HR+, Her-2 - & $19(21.1)$ \\
\hline $\mathrm{HR}+, \mathrm{Her}-2+$ & $20(22.2)$ \\
\hline Her-2 enrich & $14(15.6)$ \\
\hline Her-2 positive & $34(37.7)$ \\
\hline TNBC & $13(14.4)$ \\
\hline \multicolumn{2}{|l|}{ Initial stage at diagnosis } \\
\hline I & $11(12.2)$ \\
\hline II & $25(27.8)$ \\
\hline III & $16(17.7)$ \\
\hline IV & $38(42.2)$ \\
\hline \multicolumn{2}{|l|}{ Metastatic site at vinorelbine } \\
\hline Lung & $45(50.0)$ \\
\hline Liver & $28(31.1)$ \\
\hline Brain & $8(7.8)$ \\
\hline Bone only & $9(10.0)$ \\
\hline Skin/Soft tissue & $11(12.2)$ \\
\hline Others & $12(13.3)$ \\
\hline \multicolumn{2}{|l|}{ Number of metastatic sites } \\
\hline 1 & $42(46.7)$ \\
\hline 2 & $22(24.4)$ \\
\hline$\geq 3$ & $26(28.9)$ \\
\hline \multicolumn{2}{|l|}{ Previous treatment for MBC } \\
\hline 0 & $10(11.1)$ \\
\hline 1 & $49(54.4)$ \\
\hline 2 & $17(18.9)$ \\
\hline$\geq 3$ & $14(15.6)$ \\
\hline \multicolumn{2}{|l|}{ Response to previous treatment } \\
\hline $\mathrm{CR}$ & $1(1.1)$ \\
\hline PR & $45(50.0)$ \\
\hline SD & $14(15.6)$ \\
\hline PD & $26(28.9)$ \\
\hline $\mathrm{NE}$ & $4(4.4)$ \\
\hline
\end{tabular}

\section{Treatment efficacy}

The ORR was $53.3 \%$ and overall DCR was $78.9 \%$ for all patients; 4 (4.4\%) patients achieved CR, 44 (48.9\%) achieved PR, and 23 (25.6\%) had SD (Table 2). Almost all molecular subtypes had a good response

$\mathbf{A}$

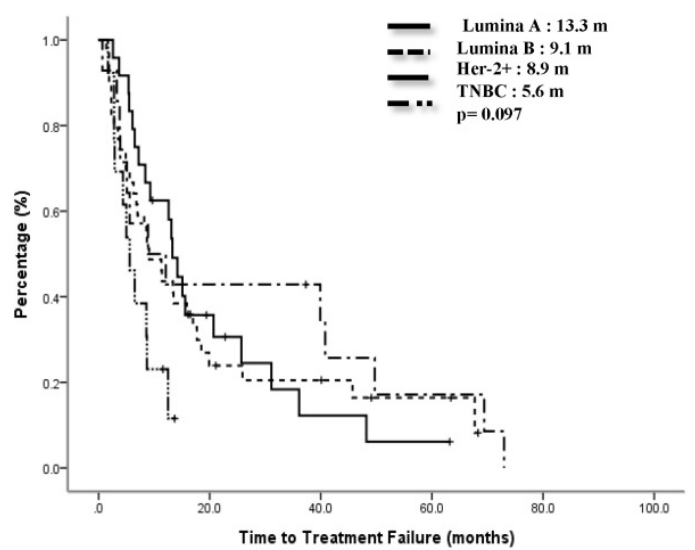

except 13 cases of TNBC. Overall response rate for HR+/Her-2 -, Her-2 + (including HR+/Her-2 + and Her- 2 enrich) and TNBC were $60.5 \%, 58.8 \%$ and $7.7 \%$ respectively. Disease control rate for $\mathrm{HR}+/ \mathrm{Her}-2$-, Her- $2+$ and TNBC were $88.4 \%, 82.3 \%$ and $30.8 \%$. TNBC group was significantly worse than other groups $(\mathrm{p}<0.001)$ (Table 2$)$.

The median TTF of the Lumina A patients was 13.3 months, Lumina B patients was 9.1 months, Her-2 enrich patients was 8.9 months, and TNBC patients was 5.6 months. Compared to the TNBC group, the non-TNBC group had a trend toward better median TTF, $p=0.097$ (Figure 1A). Median OS for Lumina A, Lumina B, Her-2 enrich and TNBC were 54.6 months, 53.3 months, 59.5 months and 24.5 months separately, $\mathrm{p}=0.28$ (Figure 1B). If we divided patients into 3 groups, the median TTF for HR+/Her-2-, Her-2+ and TNBC were 13.3 months, 8.9 months and 5.6 months, $\mathrm{p}=0.048$ (Fig 2A), median OS were 54.2 months, 69.4 months and 24.5 months separately, $\mathrm{p}=0.121$ (Figure 2B).

Previous treatments might have impacts on the effect of mVNR. For 46 patients whose treatment response to the previous regimen were $\mathrm{CR}$ or $\mathrm{PR}$, the ORR to mVNR was $65.5 \%$ and DCR was $80.4 \%$; for 14 patients whose previous treatment response was SD, the ORR to mVNR was $42.9 \%$ and DCR was $92.9 \%$; and for 26 patients whose previous treatment response was $\mathrm{PD}$, the ORR to $\mathrm{mVNR}$ was $46.2 \%$ and DCR was $65.4 \%(p=0.197)$ (Table 3). Median TTF for previous treatment response was CR/PR, SD, PD and NE were 12.5 months, 15.1 months, 6.5 months and 7.2 months, $\mathrm{p}=0.015$ (Fig 3A) and median OS were 69.4 months, 53.4 months, 41.5 months, and 32.0 months separately, $\mathrm{p}=0.135$ (Figure 3B).

B

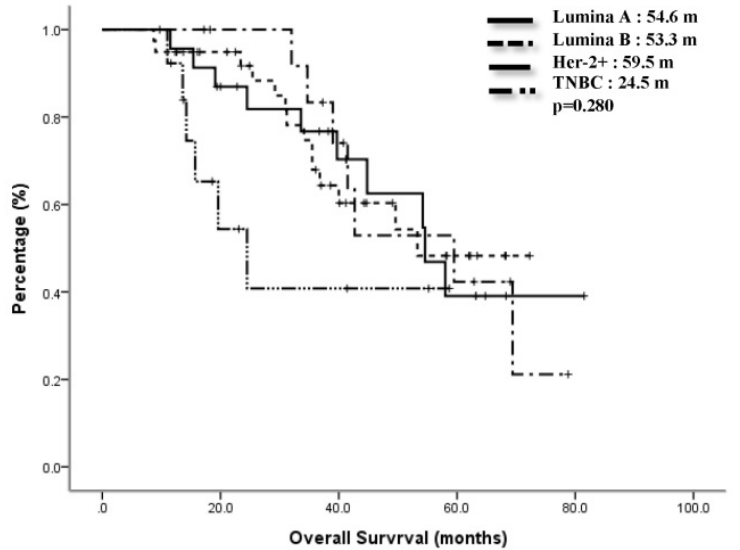

Figure 1. Time to treatment failure (A) and overall survival (B) of all 4 subgroups. 
Table 2. Best treatment response in different populations.

\begin{tabular}{|c|c|c|c|c|c|c|}
\hline Tumor response & CR, n (\%) & PR, n (\%) & SD, n (\%) & DCR, n (\%) & PD, n (\%) & $\mathrm{NE}, \mathrm{n}(\%)$ \\
\hline Overall, $\mathrm{N}=90$ & $4(4.4)$ & $44(48.9)$ & $23(25.6)$ & $71(78.9)$ & $17(18.9)$ & $2(2.2)$ \\
\hline \multicolumn{7}{|l|}{ Subtypes } \\
\hline Lumina A, N=24 & $2(8.3)$ & $15(62.5)$ & $6(25.0)$ & $23(95.8)$ & $1(4.2)$ & 0 \\
\hline Lumina B, HR+, Her-2-, N=19 & 0 & $9(47.4)$ & $6(31.6)$ & $15(78.9)$ & $3(15.8)$ & $1(5.2)$ \\
\hline Lumina $\mathrm{B}, \mathrm{HR}+, \mathrm{Her}-2+, \mathrm{N}=20$ & 0 & $12(60.0)$ & $5(25.0)$ & $17(85.0)$ & $3(15.0)$ & 0 \\
\hline Her-2 enrich , $N=14$ & $2(14.3)$ & $8(57.1)$ & $3(21.4)$ & $13(92.9)$ & $1(7.1)$ & 0 \\
\hline HR,+ Her $-2-, N=43$ & $2(4.7)$ & $24(55.8)$ & $12(27.9)$ & $38(88.4)$ & $4(9.3)$ & $1(2.3)$ \\
\hline Her-2+, N=34 & $2(5.9)$ & $18(52.9)$ & $8(23.5)$ & $28(82.3)$ & $4(11.8)$ & $1(2.9)$ \\
\hline TNBC, $N=13$ & 0 & $1(7.7)$ & $3(23.1)$ & $4(30.8)$ & $9(69.2)$ & 0 \\
\hline
\end{tabular}

CR-complete response; PD-progressive disease; PR-partial response; SD-stable disease; DCR-disease control rate; NE-not evaluable, HR-hormonal receptor; TNBC-triple negative breast cancer.

Table 3. Comparisons of previous treatment response and the best treatment response to $\mathrm{mVNR}(\mathrm{N}=90)$.

\begin{tabular}{|c|c|c|c|c|c|c|}
\hline Response to previous treatment & CR, n (\%) & PR, n (\%) & SD, n (\%) & DCR, n (\%) & PD, n (\%) & NE, $n(\%)$ \\
\hline CR and $P R, n=46$ & $1(3.4)$ & $26(62.1)$ & $10(24.1)$ & $37(80.4)$ & $8(3.4)$ & $1(6.9)$ \\
\hline $\mathrm{SD}, \mathrm{n}=14$ & 0 & $6(42.9)$ & $7(50.0)$ & $13(92.9)$ & $1(7.1)$ & 0 \\
\hline$P D, n=26$ & 0 & $12(46.2)$ & $5(19.2)$ & $17(65.4)$ & $8(30.8)$ & $1(3.8)$ \\
\hline $\mathrm{NE}, \mathrm{n}=4$ & $1(25)$ & $2(50)$ & $1(25)$ & $4(100)$ & 0 & 0 \\
\hline
\end{tabular}

CR-complete response; PD-progressive disease; PR-partial response; SD-stable disease; DCR-disease control rate; NE-not evaluable.

$\mathbf{A}$

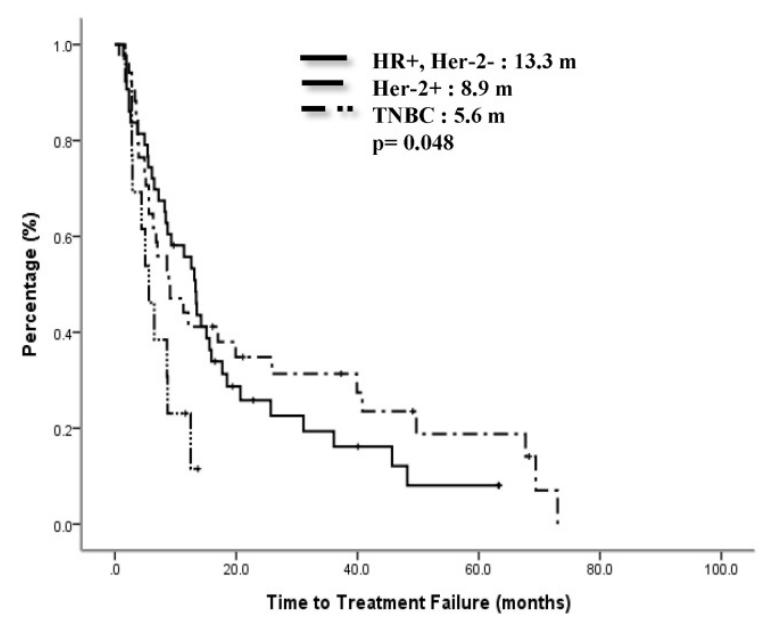

B

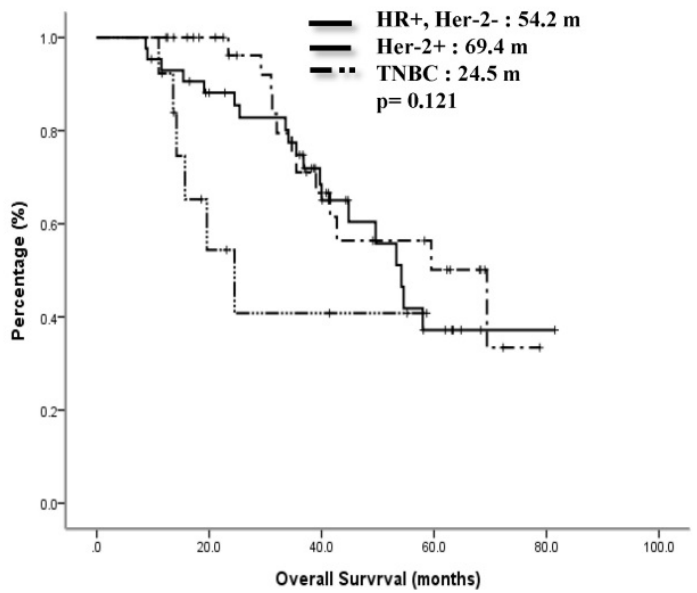

Figure 2. Time to treatment failure (A) and overall survival (B) of 3 groups.

A

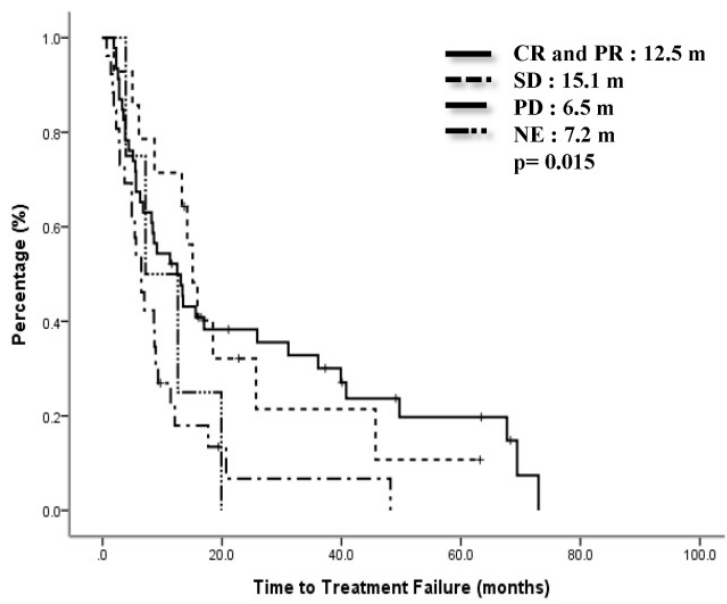

B

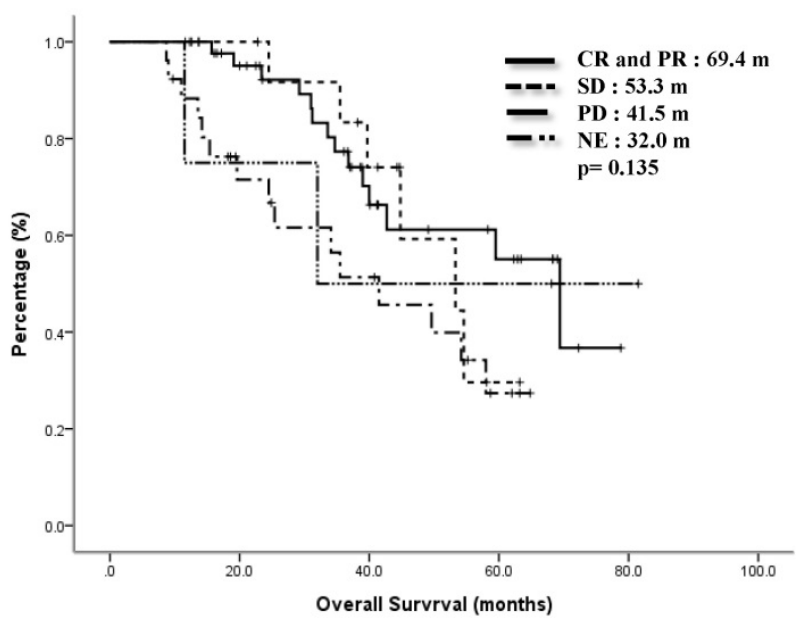

Figure 3. Time to treatment failure $(A)$ and overall survival $(B)$ by response to previous treatment. 
A

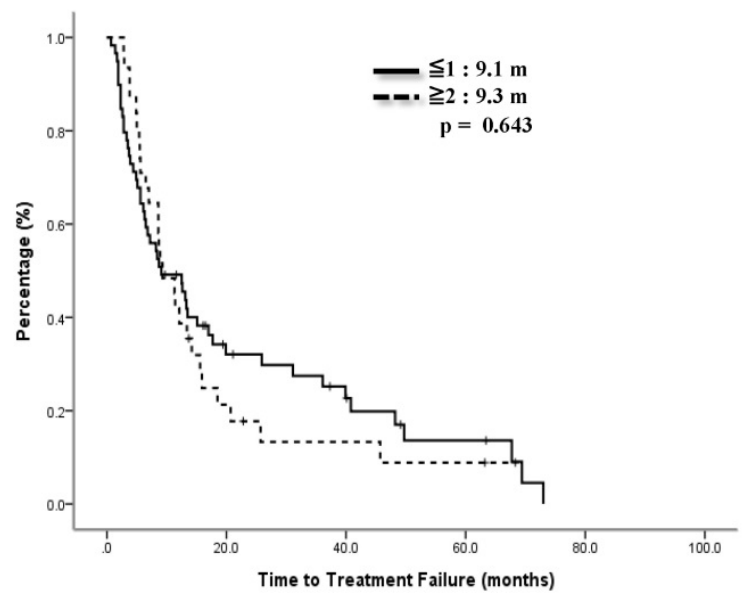

B

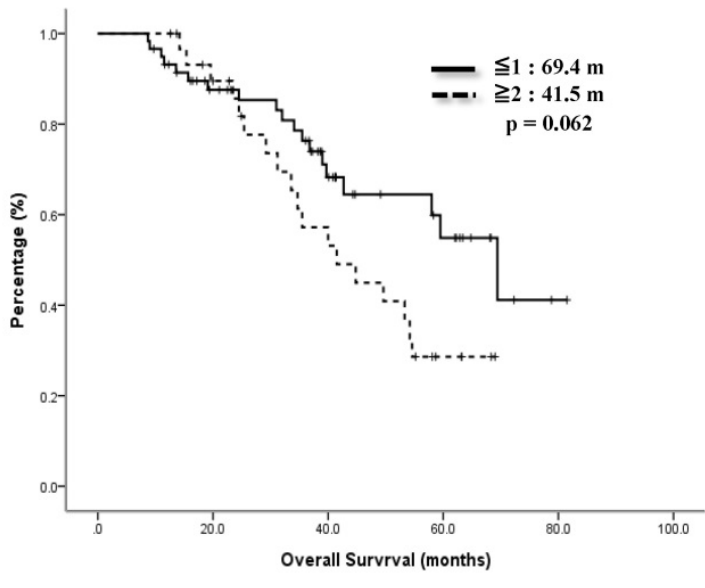

Figure 4. Time to treatment failure $(A)$ and overall survival $(B)$ by treatment linage of vinorelbine.

Table 4. Comparisons of line of treatment and best treatment response with $m V N R(N=90)$.

\begin{tabular}{llllll}
\hline Linage of previous treatments & CR, $\mathbf{n}(\%)$ & PR, $\mathbf{n}(\%)$ & SD, $\mathbf{n}(\%)$ & DCR, $\mathbf{n}(\%)$ & PD, $\mathbf{n}(\%)$ \\
\hline$\leq 1, N=59$ & $3(4.9)$ & $30(56.1)$ & $12(22.0)$ & $45(83.0)$ & $12(12.2)$ \\
$\geq 2, N=31$ & $1(3.2)$ & $14(45.1)$ & $11(35.5)$ & $26(83.9)$ & $5(16.1)$
\end{tabular}

CR-complete response; PD-progressive disease; PR-partial response; SD-stable disease; DCR-disease control rate; NE-not evaluable.

Table 5. Side effects from mVNR n (\%).

\begin{tabular}{|c|c|c|c|c|}
\hline & Gr I & Gr II & Gr III & Gr IV \\
\hline Nausea/Vomiting & $20(22.2)$ & $16(17.8)$ & 0 & 0 \\
\hline Diarrhea & 14 (15.6) & $7(7.8)$ & $1(1.1)$ & 0 \\
\hline Neutropenia & $5(5.6)$ & $5(5.6)$ & 0 & 0 \\
\hline Anemia & $11(12.2)$ & $5(5.56)$ & $1(1.1)$ & 0 \\
\hline Paresthesia & $1(1.1)$ & $2(2.2)$ & 0 & 0 \\
\hline
\end{tabular}

mVNR-metronomic vinorelbine; Gr-grade.

We also checked the impact of linage of mVNR on therapeutic effects. $59(65.6 \%)$ cases took mVNR as the first or secondary line of therapy, ORR was $61.0 \%$ and DCR was $83.0 \%$, including 3 cases of CR. Another $31(34.4 \%)$ cases who tool $\mathrm{mVNR}$ after $2^{\text {nd }}$ line of therapy, ORR was $48.3 \%$ and DCR was $83.9 \%$. Although more patients got response to $\mathrm{mVNR}$ $(61.0 \%$ vs $48.3 \%)$ as the early line, DCR were the same between early and later lines of $\mathrm{mVNR}(\mathrm{p}=.499$, Table 4). The median TTF for earlier line was 9.1 months and later line was 9.3 months, $\mathrm{p}=0.643$ (Figure 4A). Median OS for earlier line was 69.4 months versus 41.5 months of the later line, $p=0.062$ (Figure 4B).

Most patients did not have prominent side effects. The most common side effect was nausea/vomiting, followed by diarrhea and anemia. Leukopenia was not common (Table 5). A large proportion of the patients did not require pre-medications finally. Only 2 patients discontinued mVNR because of side effects. Other patients stopped treatment because of disease progression.

\section{Discussion}

The basic definition of MCT is constant administration of chemotherapy at a low, minimally toxic doses with no prolonged drug-free breaks [14]. A meta-analysis of the efficacy and toxicities of MCT for $\mathrm{ABC}$, consisted of 22 clinical trials with 1,360 patients, the pooled ORR was $34.1 \%$, CBR was $55.6 \%$. The overall 6-month PFS, 12-month OS, and 24-month OS rates were $56.8 \%, 70.3 \%$, and $40.0 \%$, respectively. The pooled incidence of grade $3 / 4$ AEs was $29.5 \%$. Conclusion of this meta-analysis was MCT may be a promising therapeutic method for MBC patients, with a favorable tumor response, survival rate, and low toxicity profile [15].

As an agent of antimicrotubule, vinorelbine can affect adhesion and tight junction of endothelium, intracellular transport of proteins and vesicles, also cell shape and cell polarization. It can also block signaling pathway of vascular endothelium growth factor. Therefore, mVNR can interfere with proliferation and migration of endothelial cells, and inhibit tube formation, sprouting and maintenance of 
the tumor vasculature and angiogenesis in addition to anti-cancer effect [16]. In our practice, we choose oral VNR as the drug for MCT instead of CAP or CTX. The major reason is the flexibility of dosage adjustment and scheduling; patients do not have to take the drug everyday like CTX or CAP $[17,18]$.

In our study, the ORR of mVNR for all patients was $65.5 \%$, the overall DCR was $80.4 \%$, and the median TTF ranged from 5.6 months for TNBC to 13.3 months for Lumina A. Compared with the results of clinical trials with highly selected patients where the ORR ranged from $17 \%$ to $62 \%$, DCR ranged from $24 \%$ to $75 \%$, and median PFS was from 3.8 months to 9.82 months, our real world outcome was only inferior to some combination therapy-based MCTs [14]. There were no significant differences of median TTF within subtypes, except for TNBC patients. This subtype was the most important risk factor for worse PFS and OS, especially for single agent MCT [19].

In contrast to TNBC, ORR of HR+/Her-2- ABC in our study was $60.5 \%$ (Table 2), TTF was 13.3 months and the OS was 54.2 months (Fig. 2). All these patents had combined at least one anti-hormonal agent. Almost in all guidelines for breast cancer treatment, hormonal therapy is not recommended to be combined with chemotherapy because of the possible negative impact on therapeutic effects to each other and no survival benefit [20]. Several studies had confirmed that combination of endocrine therapy with MCT were active for MBC [21-23]. The targets of metronomic chemotherapy are not only tumor cells but also their microenvironment, especially tumor's neovascularization [6]. In SOLTI-1501 VENTANA window of opportunity trial, Adamo et al checked the biological effect of mVNR alone or with letrozole for patients with early breast cancers. They found 3-weeks neoadjuvant mVNR combined with letrozole had superior anti-proliferative effect than both monotherapies. They also found that $\mathrm{mVNB}$ differentiated tumor cells into a slightly more estrogen-dependent state, in this context, letrozole was more effective [24].

Currently the choices of secondary line treatment for $\mathrm{HR}+/ \mathrm{Her}-2$ - depend on the previous treatments. In PALOMA3, palbociclib combined with fulvestrant, the ORR was $19 \%$, CBR was $67 \%$ [25], updated median PFS was 11.2 months and median OS was 34.9 months [26]. In MONARCH-2, abemaciclib combined with fulvestrant, the ORR was $48.1 \%$ [27], updated median PFS was 16.9 months, and the median OS was 46.7 months [28]. In MONALEESA-3, ribociclib combined with fulvestrant, median PFS was 14.6 months and median OS was 40.2 months [29]. Comparing to CDK4/6 inhibitors combine with fulvestrant as the secondary line therapy, therapeutic effects of mVNR combined with one anti-hormonal therapy are not inferior to published data, $\mathrm{mVNR}$ also has fewer side effects, especially leukopenia, and the price is less expensive than CDK4/6 inhibitor/ fulvestrant combination. This issue is more important for patients at developing countries.

Most of time, in the metronomic studies, they chose HR positive tumors, indolent disease, and bone metastases only diseases, Her-2 + ABCs are excluded. In fact, VNR combined with trastuzumab has been shown to be at least as effective as docetaxel and trastuzumab as the first-line therapy of Her-2+ ABC, and has significantly fewer adverse effects [30]. In the CLEOPATRA study, patients in each group received docetaxel for a median of 8 cycles [31]. The median OS was 57.1 months (95\% CI 50-72) in the dual blockade group and 40.8 months (36-48) in the placebo group (hazard ratio 0.69, 95\% CI 0.58-0.82) [32]. Although trastuzumab/pertuzumab and a taxane-based combination regimen is now the backbone for Her- $2+$ MBCs, most patients still have to stop taxane because of cumulative toxicities [31]. Once chemotherapy is stopped and only anti-Her-2 antibodies are administered, development of resistance is common, especially for HR-negative ABCs. It is reasonable to use $\mathrm{mVNR}$ in combination with trastuzumab as a switch maintenance therapy under this situation. Compared with conventional weekly administration, mVNR can achieve the same efficacy but with much fewer side effects. Recently, a phase II study focused on older and frail population with Her-2+ patients of $A B C$ reported that dual blockade of Her-2 plus metronomic chemotherapy provided a better PFS than Her-2 dual blockade alone, and has an acceptable safety profile [33]. Fadi et al. reported a phase II study in which oral VNR was given weekly in combination with trastuzumab as the first-line therapy of Her-2+ $\mathrm{ABC}$ [34]. In their study, the DCR was $88 \%$ and the median PFS was 6.7 month, but grade $3 / 4$ hematological toxicities including neutropenia (46\%), anemia (4\%), and nausea/vomiting (11.5\%) were observed.

Our data showed that as a switch maintenance, the ORR of mVNR in Her-2+ group reached 58.8\%, DCR was $82.3 \%$, the median TTF was 8.9 months and the median OS was 69.4 months, indicating mVNR can further extend the effect from taxane-based combination (Table 2, Figure 2). Interestingly, all our patients had received taxane before, but none exhibited cumulative neurotoxicity. Because dual blockades are not reimbursed at many countries, it is not easy for patients to receive dual blockade as a maintenance therapy, combination of mVNR with trastuzumab after taxane can be an effective and reasonable choice. 
Most of our patients had received at least one line of systemic therapy before they received mVNR. Patients whose diseases were under control by the last treatment (CR and PR) had better ORR, DCR, TTF and OS than those were with PD $(65.5 \%$ vs $46.2 \% ; 80.4 \%$ vs $65.4 \%$; 12.5 months vs 6.5 months and 68.4 months versus 41.5 months; Table 3, Fig. 3). Because most patients had stable or responsive disease when they switched to mVNR, the change was similar to a change in switching maintenance therapy. This switch did provide an alternatively effective therapy, which maintained the previous treatment response, extended the PFS and might be OS in the future, and the toxicities were minimal. The concept of switching maintenance therapy has already been commonly used in patients with lung cancer; when the cancer is under control, a change from a more toxic treatment to a less toxic treatment is made [35].

Whether the effect of mVNR is as effective as standard protocols is under evaluation. The NAME trial, which is a randomized, open-label, parallel, multi-center study, aims to evaluate the efficacy and safety of mVNR versus intermittent oral VNR in patients with Her-2- ABC, and the IBCSG 54-16 trial, a randomized phase II trial of $\mathrm{mVNR}$ plus $\mathrm{CYC}$ and CAP (VEX) versus weekly paclitaxel as the first-line or second-line treatment in patients with ER +/Her2 advanced or $\mathrm{MBC}$ are ongoing, and the results are awaited in 3-4 years.

In our data, TNBC patients had the worst outcome, the efficacy was similar to other study [36].The reason might be because of the mixed subtypes of TNBC [37]. How to improve therapeutic effects for TNBC patients become an important issue. Combination two or more drugs might help to reach better efficiency but no addictive toxicity. The best combination might be metronomic CAP and VNR with a clinical benefit rate near 50\% (VICTOR-1,2). A phase II study assessed the safety and efficacy of metronomic oral chemotherapy with VNR, CYC, and CAP in untreated metastatic TNBC patients. Twenty-two of 25 patients were evaluable for both efficacy and toxicities. The ORR was $27 \%$, CBR was $50 \%$, median TTP was 6.4 months and median OS was 18.4months. Grade $\geq 3$ adverse events were uncommon [36]. VICTOR-3 was designed to investigate the role of $\mathrm{mVNR}$, either as a single agent or in combination with metronomic CAP, in TNBC patients after an induction standard-dose CHT, as maintenance therapy is still ongoing [38].

The consensus of a workshop of Italian experts suggested that single agent mVNR can be a treatment choice for $\mathrm{HR}+/$ Her- 2 - patients with bone or soft tissue involvement, or with visceral metastases but no symptoms, or progressing after a first- or second- line endocrine therapy. The combination of mVNR with target therapy or other chemotherapy such as CAP or CYC appear to be a promising strategy, in order to maintain the benefits deriving from an all-oral regimen, and to avoid hospitalization[39].

VICTOR-6 study is the largest study reporting data of MCT from real world. Overall response rate of MCT ranged from $33.8 \%$ in first-line to $8.8 \%$ in forth-line setting. Disease control rate was from $81.5 \%$ to $54.4 \%$. Amount all regimens, VNR-based regimens had the highest ORR and DCR in first-line. Overall, median PFS was 7.2 months (95\%CI: 5.3-10.3) for VNR-single agent and 9.5 months for VNR-combination; median OS was 22.7 months (95\% CI 13.0-43.5) in VNR-single agent and 30.0 (95\% CI 26.2-34.7, HR 0.67) for VNR-combination regimens. But in this study, they excluded Her-2 + ABC cases in which subgroup $\mathrm{mVNR}$ showed an excellent result as a maintenance therapy in our study [40].

In our study, even mVNR was used at later lines, we still could see almost equally TTF between earlier or later line of therapies for advanced $\mathrm{BC}$, and the data is non-inferior to other drugs like eribulin and pegylated liposomal doxorubicin those are also have been approved after taxane and anthracycline[41, 42]. This might be because mVNR targets not only tumor cells, but also endothelial cells, which are believed to not develop resistance to chemotherapy.

Current guideline suggests MCT is a treatment option for patients not requiring rapid tumor response. Available regimens are low-dose oral CYC and methotrexate, capecitabine CAP or oral VNR-based regimens [20]. From our study and published data, MCT was associated with fewer toxicities, especially no drug-cumulative side effect which allows for long-term therapy and no need of frequent blood test. Compared with capecitabine which would still lead to skin toxicities, mVNR is lack of drug accumulation over time.

To our knowledge, this retrospective study reported the largest number of patients of Her-2 + $\mathrm{ABC}$ patients who receiving $\mathrm{mVNR}$ in the real world setting. We found $\mathrm{mVNR}$ can be an effective treatment for MBC or LABC of different molecular subtypes. It is also a successful maintenance therapy after intravenous chemotherapy. In combination with targeted therapy, mVNR might improve the therapeutic effectiveness. The side effects of $\mathrm{mVNR}$ are minimal and manageable.

\section{Conclusions}

MCT can target endothelial cells to inhibit angiogenesis, and directly kill cancer stem cells and cancer cells, and the effects can be strengthened when used in combination with targeted therapies [43]. 
From our study, metronomic VNR can be an effective treatment for $\mathrm{ABC}$, as either a switch maintenance therapy or a salvage therapy. Due to its good safety profiles, in combination with targeted therapy, other oral chemotherapy, hormonal therapy or even immunotherapy, mVNR can further improve PFS and the DCR without increasing toxicities. Side effects from $\mathrm{mVNR}$ are minimal and manageable. Further study should focus on the optimal dosage, schedule, and combination regimen.

\section{Acknowledgements}

The authors thank the "breast" cancer working group at Kaohsiung Chang Gung Memorial Hospital and E-Da Cancer Hospital for their great assistance of data reviewing and examining. The authors are indebted to all of the participants for providing the data.

\section{Availability of data and materials}

The datasets generated and/or analyzed during the current study are not publicly available due to ethical reasons. The can be made available to collaborators with a specific research agreement.

\section{Ethics approval and consent to participate}

The study design was approved by Institutional Review Board of Kaohsiung Chang Gung Memorial Hospital (IRB No. 201700595B0) and E-Da Cancer Hospital (EMRP-110-040).

\section{Authors' contributions}

Data analysis and project design: L.-CT., S.-Y.L., L.-C.K., and R.-K.M. Conception and study design: H.-M.C., S.-N.P., T.-Y.F., L.-W.C., H.-C.M, C.-C.C., W.-S.H. W.-S.C. Corrections of paper: R.-K.M.

The manuscript has been read and approved for submission by all authors. All the authors have contributed to preparing the manuscript, and no person or persons other than the authors listed have contributed significantly to its preparation.

\section{Competing Interests}

The authors have declared that no competing interest exists.

\section{References}

1. Kaklamani VG, Gradishar WJ. Endocrine Therapy in the Current Management of Postmenopausal Estrogen Receptor-Positive Metastatic Breast Cancer. Oncologist. 2017; 22(5): 507-517.

2. Gasparini G. Metronomic scheduling: the future of chemotherapy? Lancet Oncol. 2001; 2(12): 733-40.

3. Hanahan D, Bergers G, Bergsland E. Less is more, regularly: metronomic dosing of cytotoxic drugs can target tumor angiogenesis in mice. J Clin Invest. 2000; 105(8): 1045-7.

4. Klement G, Baruchel S, Rak J, et al. Continuous low-dose therapy with vinblastine and VEGF receptor-2 antibody induces sustained tumor regression without overt toxicity. J Clin Invest. 2000; 105(8): R15-24.
5. Boehm T, Folkman J, Browder T, O'Reilly MS. Antiangiogenic therapy of experimental cancer does not induce acquired drug resistance. Nature. 1997; 390(6658): 404-7.

6. Browder T, Butterfield CE, Kraling BM, et al. Antiangiogenic scheduling of chemotherapy improves efficacy against experimental drug-resistant cancer. Cancer Res. 2000; 60(7): 1878-86.

7. Romiti A, Falcone R, Roberto M, Marchettiet P. Current achievements and future perspectives of metronomic chemotherapy. Invest New Drugs. 2017; 35(3): 359-374

8. Pasquier E, Kavallaris M, Andre N. Metronomic chemotherapy: new rationale for new directions. Nat Rev Clin Oncol. 2010; 7(8): 455-65.

9. Caffo, O, Dipasquale M, Murgia V, et al. An evaluation of the pharmacokinetics and clinical use of vinorelbine for NSCLC treatment. Expert Opin Drug Metab Toxicol. 2013; 9(8): 1037-51.

10. Andre N, Carre M, Pasquier E. Metronomics: towards personalized chemotherapy? Nat Rev Clin Oncol. 2014; 11(7): 413-31.

11. Briasoulis E, Aravantinos G, Kouvataseas G, et al. Dose selection trial of metronomic oral vinorelbine monotherapy in patients with metastatic cancer: a hellenic cooperative oncology group clinical translational study. BMC Cancer. 2013; 13: 263.

12. Saridaki, Z, Malamos N, Kourakos P, et al. A phase I trial of oral metronomic vinorelbine plus capecitabine in patients with metastatic breast cancer. Cancer Chemother Pharmacol. 2012; 69(1): 35-42.

13. De Iuliis F, Salerno G, Taglieri $\mathrm{L}$, et al. On and off metronomic oral vinorelbine in elderly women with advanced breast cancer. Tumori. 2015; 101(1): 30-5.

14. Kerbel RS, Kamen BA. The anti-angiogenic basis of metronomic chemotherapy. Nat Rev Cancer. 2004; 4(6): 423-36.

15. Liu Y, Gu F, Dai X, et al. The efficacy and toxicity profile of metronomic chemotherapy for metastatic breast cancer: A meta-analysis. PLoS One. 2017; 12(3): e0173693.

16. Schwartz, EL. Antivascular actions of microtubule-binding drugs. Clin Cancer Res. 2009; 15(8): 2594-601.

17. Colleoni M, Orlando L, Sanna G, et al. Metronomic low-dose oral cyclophosphamide and methotrexate plus or minus thalidomide in metastatic breast cancer: antitumor activity and biological effects. Ann Oncol. 2006; 17(2): 232-8

18. Fedele P, marino A, Orlando L, et al. Efficacy and safety of low-dose metronomic chemotherapy with capecitabine in heavily pretreated patients with metastatic breast cancer. Eur J Cancer. 2012; 48(1): 24-9.

19. Vernieri C, Prisciandaro M, Nichetti F, et al. Oral Capecitabine-Vinorelbine is Associated with Longer Overall Survival When Compared to Single-Agent Capecitabine in Patients with Hormone Receptor-Positive Advanced Breast Cancer. Cancers (Basel). 2020; 12(3): 617

20. Cardoso F, Shimon SP, Senkus E, et al. 5th ESO-ESMO international consensus guidelines for advanced breast cancer (ABC 5). Ann Oncol. 2020; 31(12): 1623-1649.

21. Bottini A, Generali D, Brizzi MP, et al. Randomized phase II trial of letrozole and letrozole plus low-dose metronomic oral cyclophosphamide as primary systemic treatment in elderly breast cancer patients. J Clin Oncol. 2006; 24(22): 3623-8.

22. Licchetta A, Correale P, Migali C, et al. Oral metronomic chemo-hormonal-therapy of metastatic breast cancer with cyclophosphamide and megestrol acetate. J Chemother. 2010; 22(3): 201-4.

23. Schwartzberg LS, Wang G, Somer BG, et al. Phase II trial of fulvestrant with metronomic capecitabine for postmenopausal women with hormone receptor-positive, HER2-negative metastatic breast cancer. Clin Breast Cancer. 2014; 14(1): 13-9.

24. Adamo B, Bellet M, Pare' $\mathrm{L}$, et al. Oral metronomic vinorelbine combined with endocrine therapy in hormone receptor-positive HER2-negative breast cancer: SOLTI-1501 VENTANA window of opportunity trial. Breast Cancer Res. 2019; 21(1): 108e.

25. Cristofanilli M, Turner NC, Bondarenko I, et al. Fulvestrant plus palbociclib versus fulvestrant plus placebo for treatment of hormone-receptor-positive, HER2-negative metastatic breast cancer that progressed on previous endocrine therapy (PALOMA-3): final analysis of the multicentre, double-blind, phase 3 randomised controlled trial. Lancet Oncol. 2016; 17(4): 425-39.

26. Turner, NC, Slamon DJ, Ro J, et al. Overall Survival with Palbociclib and Fulvestrant in Advanced Breast Cancer. N Engl J Med. 2018; 379(20): 1926-36.

27. Sledge GW Jr, Toi M, Neven $P$, et al. MONARCH 2: Abemaciclib in Combination With Fulvestrant in Women With HR+/HER2- Advanced Breast Cancer Who Had Progressed While Receiving Endocrine Therapy. J Clin Oncol. 2017; 35(25): 2875-84.

28. Sledge GW Jr, Frenzel M. Analysis of Overall Survival Benefit of Abemaciclib Plus Fulvestrant in Hormone Receptor-Positive, ERBB2-Negative Breast Cancer-Reply. JAMA Oncol. 2020; 6(7): 1122-23.

29. Slamon DJ, Neven P, Chia S, et al. Overall Survival with Ribociclib plus Fulvestrant in Advanced Breast Cancer. N Engl J Med. 2020; 382(6): 514-24.

30. Andersson M, Lidbrink E, Bjerre Ket, et al. Phase III randomized study comparing docetaxel plus trastuzumab with vinorelbine plus trastuzumab as first-line therapy of metastatic or locally advanced human epidermal growth factor receptor 2-positive breast cancer: the HERNATA study. J Clin Oncol. 2011; 29(3): 264-71.

31. Baselga J, Corte's J, Kim SB, et al. Pertuzumab plus trastuzumab plus docetaxel for metastatic breast cancer. N Engl J Med. 2012; 366(2): 109-19. 
32. Swain SM, Miles D, Kim SB, et al. Pertuzumab, trastuzumab, and docetaxel for HER2-positive metastatic breast cancer (CLEOPATRA): end-of-study results from a double-blind, randomised, placebo-controlled, phase 3 study. Lancet Oncol. 2020; 21(4): 519-30.

33. Wildiers H, Tryfonidis K, Dar Largo L, et al. Pertuzumab and trastuzumab with or without metronomic chemotherapy for older patients with HER2-positive metastatic breast cancer (EORTC 75111-10114): an open-label, randomised, phase 2 trial from the Elderly Task Force/Breast Cancer Group. Lancet Oncol. 2018; 19(3): 323-36.

34. Farhat F, Kattan JG, Ghosn M. Oral vinorelbine in combination with trastuzumab as a first-line therapy of metastatic or locally advanced HER2-positive breast cancer. Cancer Chemother Pharmacol. 2016; 77(5): 1069-77.

35. Cappuzzo F, Ciuleanu T, Stelmakh L, et al. Erlotinib as maintenance treatment in advanced non-small-cell lung cancer: a multicentre, randomised, placebo-controlled phase 3 study. Lancet Oncol. 2010; 11(6): 521-9.

36. Montagna E, Bagnardi V, Cancello G, et al. Metronomic Chemotherapy for First-Line Treatment of Metastatic Triple-Negative Breast Cancer: A Phase II Trial. Breast Care (Basel). 2018; 13(3): 177-81.

37. Manjunath, M, Choudhary B. Triple-negative breast cancer: A run-through of features, classification and current therapies. Oncol Lett. 2021; 22(1): 512.

38. Cazzaniga ME, Biganzoli L, Cortesi L, et al. Treating advanced breast cancer with metronomic chemotherapy: what is known, what is new and what is the future? Onco Targets Ther. 2019; 12: 2989-97.

39. Cazzaniga ME, Camerini A, Addeo R, et al. Metronomic oral vinorelbine in advanced breast cancer and non-small-cell lung cancer: current status and future development. Future Oncol. 2016; 12(3): 373-87.

40. Cazzaniga ME, Pinotti G, Montagna E, et al. Metronomic chemotherapy for advanced breast cancer patients in the real world practice: Final results of the VICTOR-6 study. Breast. 2019; 48: 7-16.

41. Rau KM, Ou-Yang F, Chao TC, et al. Effect of eribulin on patients with metastatic breast cancer: multicenter retrospective observational study in Taiwan. Breast Cancer Res Treat. 2018; 170(3): 583-91.

42. Rau KM, Lin YC, Chen YY, et al. Pegylated liposomal doxorubicin (Lipo-Dox(R)) combined with cyclophosphamide and 5-fluorouracil is effective and safe as salvage chemotherapy in taxane-treated metastatic breast cancer: an open-label, multi-center, non-comparative phase II study. BMC Cancer. 2015; 15: 423.

43. Benzekry S, Pasquier E, Barbolosi D, et al. Metronomic reloaded: Theoretical models bringing chemotherapy into the era of precision medicine. Semin Cancer Biol. 2015; 35: 53-61. 